\begin{tabular}{cc}
\hline International Journal of Advanced Astronomy, 8(2)(2020) 29-34 \\
International Journal of Advanced Astronomy \\
SPC & Website: $w$ ww.sciencepubco.com/index.php/IJAA \\
\hline
\end{tabular}

\title{
Is Newtonian gravitational constant a quantized constant of microscopic quantum gravity?
}

\author{
U.V.S. Seshavatharam ${ }^{1 *}$, S. Lakshminarayana ${ }^{2}$ \\ ${ }^{1}$ Honorary faculty, I-SERVE, Survey no-42, Hitech city, Hyderabad-84,Telangana, India \\ ${ }^{2}$ Dept. of Nuclear Physics, Andhra University, Visakhapatnam-03,AP, India \\ *Corresponding author E-mail:seshavatharam.uvs@gmail.com
}

\begin{abstract}
Considering the Newtonian gravitational constant as a quantized constant of microscopic quantum gravity, an attempt is made to fit its value in a verifiable approach with reference to three large atomic gravitational constants pertaining to weak, strong and electromagnetic interactions linked with a quantum relation. Estimated value seems to be $865 \mathrm{ppm}$ higher than the recommended value.
\end{abstract}

Key words: Three Atomic Gravitational Constants; Newtonian Gravitational Constant; Quantum Gravity.

\section{Introduction}

Quantum gravity models [1,2] like string theory and loop quantum gravity seem to deal with generation of cosmic space-time, formation and properties of black holes, detection of gravitons, detection of tachyons and detection of extra dimensions via energy leakage. As Planck length is extremely small compared to nuclear and electroweak scales, role of quantum gravity models in understanding elementary particle physics seems to be practically insignificant.

As of now, as no model is serving to implement gravity in microscopic physics, we would like to stress the point that, the main reason for the failure of any modern unified theory is - "implementing the Newtonian gravitational constant $\left(G_{N}\right)$ as-it-is". Clearly speaking, without understanding the origin of $G_{N}$, it is impossible to construct a workable model of final unification. Our basic idea is that, $G_{N}$ is a quantized constant.

\section{Three large gravitational coupling constants}

When mass of any elementary is extremely small/negligible compared to macroscopic bodies, highly curved microscopic space-time can be addressed with large gravitational constants and magnitude of elementary gravitational constant seems to increase with decreasing mass and increasing interaction range. Based on this logic and assuming $G_{x} m_{x}^{2} \approx \hbar c$, we consider the possibility of existence of three large gravitational constants assumed to be associated with the electromagnetic, strong and weak interactions [3-6]. If so, in analogy with Planck length, it seems possible to have three different lengths corresponding to electromagnetic, strong and weak interactions.

\section{Our four basic assumptions}

We propose the following three assumptions. For details, readers are encouraged to see our old and recent papers [6-14] and references therein.

1) Each atomic interaction is associated with a characteristic gravitational constant.

2) There exists a characteristic electroweak fermion of rest energy, $M_{w} c^{2} \cong 584.725 \mathrm{GeV}$. It can be considered as the zygote of all elementary particles.

3) There exists a strong interaction elementary charge $\left(e_{s}\right)$ in such a way that, its squared ratio with normal elementary charge is close to reciprocal of the strong coupling constant.

4) Quark fermion-boson mass ratio is close to 2.27 [12-14]. 


\section{To estimate the magnitude of the Newtonian gravitational constant}

Based on the above assumptions, $G_{N}$ can be estimated in a theoretical approach with the following relations.

$$
G_{w} M_{w}^{2} \cong \hbar c
$$

Where, $G_{w}$ and $M_{w}$ represent the assumed weak interaction gravitational constant and rest mass of weak interaction fermion.

$$
m_{p} \cong\left(\frac{4 \pi^{2}}{\alpha}\right)^{\frac{1}{3}}\left(M_{w} m_{e}^{2}\right)^{\frac{1}{3}}
$$

Where, $m_{p}$ and $m_{e}$ represent the proton and electron rest masses respectively and $\alpha$ represents the fine structure ratio.

$\frac{G_{w}}{G_{N}} \cong\left(\frac{m_{p}}{m_{e}}\right)^{10}$

On simplification,

$$
G_{N} \cong \frac{16 \pi^{4}}{\alpha^{2}}\left(\frac{m_{e}}{m_{p}}\right)^{14}\left(\frac{\hbar c}{m_{p}^{2}}\right)
$$

This relation seems to be satisfied at only one value of $G_{N}$. Obtained value is $G_{N} \cong 6.679855429 \times 10^{-11} \mathrm{~m}^{3} \mathrm{~kg}^{-1} \mathrm{sec}^{-2}$ and its CODATA 2014 recommended value [15], [16] is $G_{N} \cong 6.67408 \times 10^{-11} \mathrm{~m}^{3} \mathrm{~kg}^{-1} \mathrm{sec}^{-2}$. Error is around $865 \mathrm{ppm}$. It needs further study with respect to ongoing experiments [17-20]. Another interesting relation is,

$$
G_{N} \cong \frac{G_{w}^{21} G_{e}^{10}}{G_{s}^{30}}
$$

$$
\text { where }\left\{\begin{array}{l}
G_{w} \cong 2.909745179 \times 10^{22} \mathrm{~m}^{3} \mathrm{~kg}^{-1} \mathrm{sec}^{-2} \\
G_{s} \cong 3.329560557 \times 10^{28} \mathrm{~m}^{3} \mathrm{~kg}^{-1} \mathrm{sec}^{-2} \\
G_{e} \cong 2.374335306 \times 10^{37} \mathrm{~m}^{3} \mathrm{~kg}^{-1} \mathrm{sec}^{-2}
\end{array}\right\}
$$

where subscripts $(w, s$ and $e$ ) refer to weak, strong and electromagnetic interactions.

\section{To validate the recommended value of the Newtonian gravitational constant}

Considering the recommended value of $G_{N}$,

1) Based on relation (3), value of $G_{w}$ can be estimated.

2) By inserting the value of $G_{w}$ in relation (1), value of $M_{w}$ can be estimated.

3) By inserting the value of $M_{w}$ in relation (2), value of $m_{p}$ can be estimated.

4) By comparing the estimated value of $m_{p}$ with its recommended value, given value of $G_{N}$ can be validated.

5) With reference to the recommended value of $G_{N} \cong 6.67408 \times 10^{-11} \mathrm{~m}^{3} \mathrm{~kg}^{-1} \mathrm{sec}^{-2}$, estimated value of $m_{p}$ seems to be $144 \mathrm{ppm}$ higher than the recommended value of $m_{p}$.

6) In this way, recommended value of $G_{N}$ can be validated.

\section{About the Fermi's weak coupling constant}

Based on relation (1), it is natural to expect a relation for estimating the Fermi's weak coupling constant $G_{F}$. We noticed that,

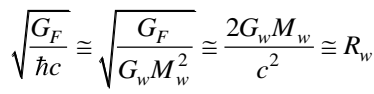




$$
\begin{aligned}
\therefore \quad G_{F} & \cong G_{w} M_{w}^{2} R_{w}^{2} \cong \frac{4 G_{w}^{3} M_{w}^{4}}{c^{4}} \\
& \cong 1.440210094 \times 10^{-62} \mathrm{~J} . \mathrm{m}^{3}
\end{aligned}
$$

This value is around $3000 \mathrm{ppm}$ higher than the recommended value of $G_{F} \cong 1.43589 \times 10^{-62} \mathrm{~J} \cdot \mathrm{m}^{3}$.It needs further study [15], [16].

\section{A new definition for the strong coupling constant}

Based on our assumption-3, strong coupling constant can be defined as,

$$
\begin{aligned}
& \frac{e_{s}}{e} \cong \frac{G_{s} m_{p}^{2}}{\hbar c} \cong \frac{G_{s} m_{p}^{2}}{G_{w} M_{w}^{2}} \\
& \cong \sqrt{\frac{1}{\alpha_{s}}} \cong \sqrt{\frac{1}{0.1151937}} \cong 2.9463591
\end{aligned}
$$

Where, $e_{s}$ and $e$ represent the strong nuclear charge and elementary charge respectively and $\alpha_{s}$ represents the strong coupling constant.

$$
\begin{aligned}
& \left(\frac{e_{s}}{e}\right)^{2} \cong\left(\frac{G_{s} m_{p}^{2}}{\hbar c}\right)^{2} \cong\left(\frac{G_{s} m_{p}^{2}}{G_{w} M_{w}^{2}}\right)^{2} \\
& \cong \frac{1}{\alpha_{s}} \cong \frac{1}{0.1151937} \cong 8.68103
\end{aligned}
$$

Based on this concept,

1) Proton magnetic moment can be addressed with, $\mu_{p} \cong \frac{e_{s} \hbar}{2 m_{p}} \cong \frac{e G_{s} m_{p}}{2 c} \cong 1.48 \times 10^{-26} \mathrm{~J} /$ Tesla

2) Nuclear binding energy can be understood with three simple terms having a common energy coefficient[7-10], $B_{0} \cong \frac{e_{s}^{2}}{4 \pi \varepsilon_{0} R_{0}} \cong\left(\frac{e e_{s}}{8 \pi \varepsilon_{0} \hbar c}\right) m_{p} c^{2} \cong 10.1 \mathrm{MeV}$ where $R_{0} \cong \frac{2 G_{s} m_{p}}{c^{2}} \cong 1.24 \mathrm{fm}$.

3) For medium and heavy atomic nuclides, nuclear charge radii can be addressed with, $R_{(Z, A)} \cong(Z+\sqrt{Z(A-Z)})^{\frac{1}{3}}\left(\frac{G_{s} m_{p}}{c^{2}}\right)$ where $Z, A$ represent proton number and mass number respectively.

\section{Phase transition points of elementary particles}

With reference to Hawking's black hole temperature formula [3], [21] and based on the three atomic gravitational constants, phase transition points $\left(T_{p t}\right)$ of elementary particles can be addressed with,

$$
T_{p t} \approx \frac{\hbar c^{3}}{8 \pi k_{B}\left(G_{x} m_{x}\right)}
$$

where, $G_{x}$ and $m_{x}$ represent the interaction based characteristic gravitational constant and mass respectively. If so, for electrons, $T_{p t} \cong 3.79 \times 10^{5}{ }^{\circ} \mathrm{K}$, for protons, $T_{p t} \cong 1.47 \times 10^{11}{ }^{\circ} \mathrm{K}$ and for the new weak fermion of rest energy $584.725 \mathrm{GeV}, T_{p t} \cong 2.70 \times 10^{14}{ }^{\circ} \mathrm{K}$. Keeping the family of particles in view, above relation can be expressed as,

$$
T_{p t} \cong\left(\frac{m_{y}}{m_{x}}\right) \frac{\hbar c^{3}}{8 \pi k_{B}\left(G_{x} m_{x}\right)}
$$

Where, $m_{x}$ and $m_{y}$ represent the interaction based characteristic mass of ground state and excited state respectively. For leptons, $m_{x} \cong m_{e}$ and $m_{y} \cong m_{\mu}$ or $m_{\tau}$.

\section{Characteristic elementary forces and their corresponding potentials and amplitudes}

'String tension' is a practical aspect of String theory. Considering the proposed three atomic gravitational constants and following the universal applicability of 'speed of light', approximate tensions [22] associated with weak, strong, electromagnetic and gravitational interactions can be represented by, 


$$
\left\{\begin{array}{l}
F_{w} \cong\left(\frac{c^{4}}{4 G_{w}}\right) \cong 6.94 \times 10^{10} \mathrm{~N} \\
F_{s} \cong\left(\frac{c^{4}}{4 G_{s}}\right) \cong 6.065 \times 10^{4} \mathrm{~N} \\
F_{e} \cong\left(\frac{c^{4}}{4 G_{e}}\right) \cong 8.505 \times 10^{-5} \mathrm{~N} \\
F_{g} \cong\left(\frac{c^{4}}{4 G_{N}}\right) \cong 3.026 \times 10^{43} \mathrm{~N}
\end{array}\right\}
$$

Following the universal applicability of 'elementary charge', approximate (operating) energy potentials associated with above string tensions can be represented by,

$$
\left\{\begin{array}{l}
E_{w} \cong \sqrt{\frac{e^{2}}{4 \pi \varepsilon_{0}}\left(\frac{c^{4}}{4 G_{w}}\right)} \cong 25.0 \mathrm{GeV} \\
E_{s} \cong \sqrt{\frac{e^{2}}{4 \pi \varepsilon_{0}}\left(\frac{c^{4}}{4 G_{s}}\right)} \cong 23.3 \mathrm{MeV} \\
E_{e} \cong \sqrt{\frac{e^{2}}{4 \pi \varepsilon_{0}}\left(\frac{c^{4}}{4 G_{e}}\right)} \cong 874 \mathrm{eV} \\
E_{g} \cong \sqrt{\frac{e^{2}}{4 \pi \varepsilon_{0}}\left(\frac{c^{4}}{4 G_{N}}\right)} \cong 8.355 \times 10^{7} \mathrm{~J}
\end{array}\right\}
$$

These estimated weak, strong and electromagnetic energy potentials seem to be close to experimental values. Corresponding string amplitudes can be expressed as follows.

$$
\left\{\begin{array}{l}
L_{w} \cong \sqrt{\frac{G_{w} \hbar}{c^{3}}} \cong 3.375 \times 10^{-19} \mathrm{~m} \\
L_{s} \cong \sqrt{\frac{G_{s} \hbar}{c^{3}}} \cong 3.61 \times 10^{-16} \mathrm{~m} \\
L_{e} \cong \sqrt{\frac{G_{e} \hbar}{c^{3}}} \cong 9.64 \times 10^{-12} \mathrm{~m} \\
L_{g} \cong \sqrt{\frac{G_{N} \hbar}{c^{3}}} \cong 1.617 \times 10^{-35} \mathrm{~m}
\end{array}\right\}
$$

\section{Astrophysical applications of electromagnetic, strong and weak gravitational constants}

\subsection{Observing $3.5 \mathrm{keV}$ galactic photon}

Recent galactic X-ray studies [23] strongly confirmed the existence of a new photon of energy $3.5 \mathrm{keV}$. So far, its origin is unknown and unclear. In this context, we proposed [9] the following alternative mechanism for understanding the origin of $3.5 \mathrm{keV}$ photon.

1) At higher temperatures, astrophysical objects generate charged leptons of rest mass $\left(m_{x l}\right)^{ \pm} \cong \sqrt{\frac{e^{2}}{4 \pi \varepsilon_{0} G_{e}}} \cong 1.75 \mathrm{keV} / \mathrm{c}^{2}$.

2) With pair annihilation mechanism, $\left(m_{x l}\right)$ generates a photon of rest energy $3.5 \mathrm{keV}$.

3) Based on $1.75 \mathrm{keV}$ lepton, muon and tau rest masses can be fitted with a formula [9],

$$
\left.m_{(\mu, \tau)} c^{2} \cong\left[\gamma^{3}+\left(n^{2} \gamma\right)^{n}\left(\frac{G_{e}}{G_{N}}\right)^{1 / 4}\right]^{\frac{1}{3}} 1.75 \mathrm{keV}\right\} \cong(106.5,1781.5) \mathrm{MeV} \text { where, } \gamma \cong \sqrt{\frac{4 \pi \varepsilon_{0} G_{e} m_{e}^{2}}{e^{2}}} \cong 292.187 \text { and } n=1 \text { and } 2 \text {. }
$$

\subsection{Observing TeV galactic photons}

If one is willing to consider any massive astrophysical object as a blending of $M_{w} c^{2} \cong 584.725 \mathrm{GeV}$ fermions, at higher temperatures, there is scope for observing photons of energy $1.17 \mathrm{TeV}$ with pair annihilation mechanism of $\left(M_{w}\right)^{ \pm}$. Proceeding further, (like electrons) considering inverse Compton effect associated with $M_{c^{2}}$, photons of energy of the order TeV can also be understood easily [24].

\subsection{Stellar black hole mass limit}

Astrophysics point of view, by considering the combination of nuclear gravitational constant and Newtonian gravitational constant, i.e. $\left(G_{s}, G_{N}\right)$, there is a scope for understanding stellar black hole mass limit [9], [25].Assuming a stellar black hole of mass density equals to 
nuclear density, it is possible to show that, $\frac{G_{N} M_{B}}{G_{s} m_{n}} \cong \sqrt{\frac{G_{s}}{G_{N}}}$, where $\left(M_{B}, m_{n}\right)$ represent black hole and nucleon mass respectively and nuclear radius, $R_{0} \cong \frac{2 G_{s} m_{n}}{c^{2}} \cong 1.24 \mathrm{fm}$. Thus characteristic mass limit of a stellar black hole can be estimated to be, $M_{B} \cong\left(\frac{G_{s}}{G_{N}}\right)^{\frac{3}{2}}\left(m_{n}\right) \cong 9.37$ solar masses.

Point to be noted is that, mass of stellar black hole increases with decreasing density. Clearly speaking,

1) If density of stellar black hole is less than the nuclear density, its mass seems to be higher than 9.37 solar masses.

2) If density of stellar black hole is greater than the nuclear density, its mass seems to be smaller than 9.37 solar masses.

3) Nuclear density can be considered as a characteristic reference of stellar black hole density.

4) In a simplified view, $\frac{\text { Black hole surface area }}{\text { Nuclear surface area }} \cong\left(\frac{G_{s}}{G_{N}}\right)$.

\subsection{Stellar magnetic dipole moments}

With reference to strong and weak gravitational constants, stellar magnetic dipole moments can be understood [9], [26]. Considering relatively strong and relatively weak structural bonding of stellar objects of mass $M_{X}$,

i) If any stellar object is relatively strongly bound, then it can have a maximum dipole magnetic moment and can be expressed by $\left(\frac{e G_{s} M_{x}}{2 c}\right)$.

ii) If any stellar object is relatively weakly bound, then it can have a minimum dipole magnetic moment and can be expressed by $\left(\frac{e G_{w} M_{x}}{2 c}\right)$.

iii) To a very rough approximation, mean magnetic dipole moment can be approximated with a relation of the form, $\left(\frac{e \sqrt{G_{w} G_{s}} M_{x}}{2 c}\right)$

iv) Earth's estimated mean magnetic dipole moment is $4.986 \times 10^{22}$ A.m ${ }^{2}$ and actual value $8 \times 10^{22}$ A.m ${ }^{2}$.

v) Sun's, estimated mean magnetic dipole moment is $1.65 \times 10^{28} \mathrm{~A} \cdot \mathrm{m}^{2}$ and actual value $3.5 \times 10^{29}$ A.m ${ }^{2}$.

vi) With reference to other stellar compact objects and black holes, further study can be carried out.

\section{Discussion and conclusion}

1) Between 1971 - 1995, Tennakone, Abdus Salam, Sivaram and other physicists proposed the existence of a large nuclear gravitational constant [3,4]. In 2013, Roberto Onofrio proposed the existence of a large gravitational constant pertaining to weak interactions [5]. Authors' proposed model is first of its kind with a simultaneous existence of four gravitational constants pertaining to the four basic physical interactions and needs a careful study at fundamental level. Its success seems to depend on the following three points.

a) Experimental verification of weak fermion of rest energy $M_{w} c^{2} \cong 584.725 \mathrm{GeV}$.

b) Experimental verification of strong nuclear charge, $e_{s} \cong 2.9463591 e$.

c) Logical confirmation of the three atomic gravitational constants.

2) Scientists strongly believe that, many of the open questions to be addressed in any theory of quantum gravity are connected with the temperature and the entropy of black holes. Here we would like to highlight the point that, by considering the three atomic gravitational constants,

a) With reference to Hawking's black hole temperature formula, it is possible to study the phase transition temperatures of all elementary particles which in turn may help in understanding their cosmological origin.

b) With reference to Planck length formula, it is possible to study the working lengths of the three atomic interactions.

c) Testable and verifiable predictions, astrophysical observations, and particle and nuclear experiments can be carried out for all interactions in an understandable approach.

3) Point to be noted is that, either with reference to string theory or with reference to loop quantum gravity, magnitude of $G_{N}$ cannot be estimated. It was clearly mentioned by senior scientists that, as of now, there exists no relation between atomic physical constants and the Newtonian gravitational constant. In this context, our proposed method helps in understanding the basic interactions in terms of 'gravity' and also helps in estimating the magnitude of $G_{N}$.

4) Proceeding further, an in-depth research can be carried out at fundamental level.

\section{Acknowledgements}

Author Seshavatharam is indebted to professors shri M. Nagaphani Sarma, Chairman, shri K.V. Krishna Murthy, founder Chairman, Institute of Scientific Research in Vedas (I-SERVE), Hyderabad, India and Shri K.V.R.S. Murthy, former scientist IICT (CSIR), Govt. of India, Director, Research and Development, I-SERVE, for their valuable guidance and great support in developing this subject.

\section{References}

[1] Sunil Mukhi. (2011). String theory: a perspective over the last 25 years. Class. Quant. Grav. 28153001. https://doi.org/10.1088/02649381/28/15/153001.

[2] Bojowald M. (2008). Loop quantum cosmology. Living Rev. Rel. 11, 4.https://doi.org/10.12942//rr-2008-4.

[3] Tennakone K. (1974). Electron, muon, proton, and strong gravity. Phys. Rev. D. 10:1722. https://doi.org/10.1103/PhysRevD.10.1722.

[4] Salam A, Sivaram C. (1993). Strong Gravity Approach to QCD and Confinement. Mod. Phys. Lett., A8(4), $321-326$. https://doi.org/10.1142/S0217732393000325. 
[5] Roberto Onofrio. (2013) On weak interactions as short-distance manifestations of gravity. Modern Physics Letters A 28 , 1350022. https://doi.org/10.1142/S0217732313500223.

[6] Seshavatharam UVS and Lakshminarayana S. (2015) To Validate the Role of Electromagnetic and Strong Gravitational Constants via the Strong Elementary Charge. Universal Journal of Physics and Application9(5), 216 - 225. https://doi.org/10.13189/ujpa.2015.090503.

[7] Seshavatharam UVS and Lakshminarayana S. (2017) Understanding the basics of final unification with three gravitational constants associated with nuclear, electromagnetic and gravitational interactions. Journal of Nuclear Physics, Material Sciences, Radiation and Applications 4(1),1-19. https://doi.org/10.15415/jnp.2017.42031.

[8] Seshavatharam UVS and Lakshminarayana S. (2019). On the role of four gravitational constants in nuclear structure. Mapana Journal of Sciences, 18(1), 21-45. https://doi.org/10.12723/mjs.48.2.

[9] Seshavatharam UVS and Lakshminarayana S. (2020).Implications and Applications of Fermi Scale Quantum Gravity. International Astronomy and Astrophysics Research Journal.2(1),13-30.

[10] Seshavatharam UVS and Lakshminarayana S. (2020). Significance and Applications of the Strong Coupling Constant in the Light of Large Nuclear Gravity and Up and Down Quark Clusters. International Astronomy and Astrophysics Research Journal.2(1),56-68.https://doi.org/10.20944/preprints201911.0398.v2.

[11] Seshavatharam UVS and Lakshminarayana S. (2019). On the Role of Nuclear Quantum Gravity in Understanding Nuclear Stability Range of Z = 2 to 118. J. Nucl. Phys. Mat. Sci. Rad. A. 7(1), 43-51. https://doi.org/10.15415/jnp.2019.71005.

[12] Seshavatharam UVS and Lakshminarayana S. (2010). Super Symmetry in Strong and Weak interactions. Int. J. Mod. Phys. E, 19(2),263-280. https://doi.org/10.1142/S021830131001473X.

[13] Seshavatharam UVS and Lakshminarayana S. (2011). SUSY and strong nuclear gravity in (120-160) GeV mass range. Hadronic journal, $34(3)$, 277.

[14] Seshavatharam UVS and Lakshminarayana S. (2020). 4G Model of Fractional Charge Strong-Weak Super Symmetry. International Astronomy and Astrophysics Research Journal.2(1),31-55.https://doi.org/10.20944/preprints201912.0391.v1.

[15] Mohr P. J, Newell D. B and Taylor B. N. (2014). CODATA recommended values of the fundamental constants: Rev. Mod. Phys. 88, 035009.

[16] M. Tanabashi et al. (2018).(Particle Data Group), Phys. Rev. D 98, 030001. https://doi.org/10.1103/RevModPhys.88.035009.

[17] Canuel B et al. (2018). Exploring gravity with the MIGA large scale atom interferometer. Science reports, 8:14064. https://doi.org/10.1038/s41598018-32165-z.

[18] Li, Qing et al. (2018). Measurements of the gravitational constant using two independent methods. Nature 560, 582588. https://doi.org/10.1038/s41586-018-0431-5.

[19] Junfei Wu et al. (2019). Progress in Precise Measurements of the Gravitational Constant. Ann. Phys. (Berlin) 531, 1900013. https://doi.org/10.1002/andp.201900013.

[20] Victoria Xu et al. (2019). Probing gravity by holding atoms for 20 seconds. Science,366(6466), 745-749. https://doi.org/10.1126/science.aay6428

[21] Hawking S.W. (1975). Particle creation by black holes. Commun. Math. Phys.43, 199-220. https://doi.org/10.1007/BF02345020.

[22] Gibbons G.W. (2002). The Maximum Tension Principle in General Relativity. Foundations of Physics. 32: 1891 https://doi.org/10.1023/A:1022370717626.

[23] Nico Cappelluti et al. (2018). Searching for the $3.5 \mathrm{keV}$ Line in the Deep Fields with Chandra: The 10 Ms Observations. The Astrophysical Journal. 854:179. https://doi.org/10.3847/1538-4357/aaaa68.

[24] Amenomori M et al. (2019). First detection of photons with energy beyond $100 \mathrm{TeV}$ from an astrophysical source. Phys. Rev. Lett. 123, 051101. https://doi.org/10.1103/PhysRevLett.123.051101.

[25] Grzegorz Wiktorowicz et al. (2019). Populations of stellar mass Black holes from binary systems. The Astrophysical journal. 885(1). https://doi.org/10.3847/1538-4357/ab45e6.

[26] Humphreys DR. (1984). The Creation of Planetary Magnetic Fields. Creation Research Society Quarterly. 21(3). 